CE TEXTE A ÉTÉ PUBLIÉ DANS UNE VERSION MODIFIÉE DANS UN OUVRAGE COLLECTIF : ROMAINE MALENFANT ET GUY BELLEMARE (DIR.), LA DOMINATION AU TRAVAIL : DES CONCEPTIONS TOTALISANTES À LA DIVERSIFICATION DES FORMES DE DOMINATION, QUÉBEC, PUQ, P. 99-124

\title{
LA DOMINATION DANS LE MODĖLE DE PRODUCTION DE HAUTE PERFORMANCE DANS LA GESTION DE PROJETS
}

\author{
Marie-Josée Legault, Téluq \\ Stéphanie Chasserio, École supérieure de commerce de Lille
}

\section{INTRODUCTION}

Les sociologues des professions s'intéressent depuis longtemps aux formes de domination au travail qui ne s'exercent pas dans le cadre d'un assujettissement des travailleurs à un contrôle hiérarchique autoritaire, ni dans le cadre d'une division stricte entre la conception et l'exécution du travail (Legault, 1988). Ce large corpus de travaux traite surtout des travailleurs hautement qualifiés qui sont organisés en ordres professionnels et de ceux qu'emploient les grandes bureaucraties. Or, la situation contemporaine fait émerger de nouvelles formes de domination chez ces travailleurs; nous traiterons ici de l'un de ces cas, celui de la gestion par projets dans le domaine de l'économie du savoir, plus particulièrement des services technologiques aux entreprises. Des travailleurs de diverses catégories de l'informatique étudiés ici ont en commun d'être très qualifiés, de travailler dans de petites organisations matricielles et de ne pas être regroupés en un ordre professionnel.

Martuccelli (2001) a traité de nouvelles formes de domination caractérisées par une direction qui suscite l'engagement au travail par l'adhésion aux valeurs de l'entreprise et l'intériorisation de 
ses contraintes, qui déplace le contrôle vers les individus, les équipes de travail et qui fait émerger une nouvelle figure dans l'exercice du contrôle, soit le client. De la même façon, une récente recension de travaux empiriques sur le travail (Murray, Bélanger, Giles et Lapointe, 2002) conclut à un assez vaste consensus, tant des chercheurs que des praticiens, concernant l'émergence d'un nouveau modèle général de production et de travail dit de haute performance (high performance workplace) qui se distingue par la mise en place de ces formes de domination dans le monde industriel, qui remettent en cause le modèle de régulation fordiste (Bélanger, Giles et Murray, 2002, p. 20-30).

Le texte qui suit met en évidence la pertinence du modèle de haute performance pour rendre compte de la domination chez les professionnels de l'informatique dans les entreprises de services technologiques aux entreprises (ESTEs) :

- L'organisation du travail dans ces ESTEs sollicite grandement les qualifications et les savoirs de ces travailleurs, on les valorise et on est disposé à en payer le prix.

- Les travailleurs y ont une grande autonomie et un grand pouvoir d'auto-régulation de leur travail. Toutefois, comme on transfère aux travailleurs la très grande responsabilité de la satisfaction d'un client volatile, l'autonomie prend plutôt la forme d'une injonction qui, à son tour, révèle un mode de domination propre au secteur.

- Les risques liés à l'incertitude de la production sont transférés aux travailleurs : risques économiques (sous-emploi, chômage), risques sociaux (maladie, maternité, vieillesse), risques professionnels (désuétude des connaissances, faute professionnelle).

- On peut percevoir l'existence d'un certain consensus entre les professionnels et la direction, une adhésion certaine des professionnels aux objectifs de la direction et à sa lecture des contraintes propres à l'industrie, qui garantit non seulement un engagement élevé mais aussi l'acceptation par les travailleurs du risque qui leur est transféré.

\section{PROBLÉMATIQUE}

En effet, les précédents éléments distinguent le modèle de haute performance émergent du modèle fordiste (Giles, Murray et Bélanger, 2002; Godard, 2001). Ce nouveau modèle se révèle 
utile devant l'ouverture des marchés, la concurrence mondiale, la transformation de la demande et la rapidité du progrès technologique. Les directions des entreprises, pour pouvoir répondre adéquatement aux changements de leur environnement, cherchent à rendre plus flexibles :

- leur structure organisationnelle en supprimant des niveaux hiérarchiques pour adopter une structure plus efficace et rapide dans le processus de prise de décision; entre autres, on tendra par exemple à diviser l'organisation, à atomiser certains éléments pour les comparer avec des fournisseurs externes (Bélanger, Lapointe et Lévesque, 2002);

- leur offre en délaissant la production de masse uniformisée pour proposer des produits correspondant précisément aux besoins du client;

- l'organisation du travail pour adopter des formes matricielles, par exemple le travail en équipes ou la gestion par projets;

- la main-d'œuvre en développant des formes d'emploi qui permettent de faire varier l'effectif selon leurs besoins et de diminuer leurs coûts, et/ou d'embaucher une main-d'œuvre polyvalente, pour favoriser une production de qualité et variée, car conçue sur mesure.

Les entreprises de services technologiques aux entreprises (ESTEs) représentent un spécimen typique de cette conjoncture; le marché du produit est très compétitif, car on trouve de multiples fournisseurs dans le monde. Le marché du travail l'est aussi, car la main-d'œuvre est très mobile; on y embauche des professionnels hautement qualifiés en informatique pour lesquels la demande est très grande.

Or, des problèmes inhérents à ce modèle de production de haute performance, que révèle la synthèse des études de cas, mettent en évidence de nouvelles formes de domination. Les directions ressentent notamment des tensions entre les impératifs contradictoires de l'autonomie et du contrôle des travailleurs, ainsi qu'entre qualité élevée et réduction des coûts (Bélanger, Giles et Murray, 2002; Edwards, Geary et Sisson, 2002), au point de compromettre la mobilisation du travail (Appelbaum, 2002). En conséquence des mesures prises pour résoudre ces problèmes, certaines études associent aux systèmes de haute performance une détérioration des conditions de travail, une réduction de l'autonomie dans ses dimensions autres que 
professionnelles, une intensification de l'effort provoquant une augmentation du stress et des problèmes de santé au travail, une réduction de la sécurité d'emploi, une démobilisation syndicale et, enfin, un affaiblissement de la position des travailleurs dans les rapports sociaux du travail (Appelbaum, 2002; Bélanger, Lapointe et Lévesque, 2002). De plus en plus d'entreprises visent la flexibilité, remettent en question la stabilité d'emploi (Carnoy, 2000, p. 56-104; Périlleux, 2001, p. 33-35; Rousseau et Greller, 1994) tout en exigeant plus de disponibilité, de productivité et d'effort au moment présent, en d'autres termes d'engagement, et une plus grande incertitude en résulte pour les travailleurs (DeFillippi, 2003; Linhart, 2004; Périlleux, 2001). Appelbaum ne conclut pas de sa revue des travaux que les travailleurs rejettent en majorité les changements dans l'organisation du travail; bien au contraire, certains éléments emportent un vif succès dans les enquêtes, notamment le travail en équipes (aussi : Edwards, Geary et Sisson, 2002). C'est parmi certains de ces effets qu'on retrouve de nouvelles formes de domination et l'organisation du travail dans les ESTEs nous permet de les mettre en évidence.

\section{MÉTHODE}

Les résultats présentés dans ce texte sont tirés d'une enquête portant sur la conciliation entre la vie privée et la vie professionnelle (CVP) menée auprès du personnel hautement qualifié des services technologiques aux entreprises, entre janvier 2001 et avril 2002 (professionnels et hauts gestionnaires) $)^{1}$.

Dans notre échantillon d'entreprises, on retrouve cinq organisations de la nouvelle économie et deux grandes bureaucraties plus traditionnelles servant de secteur de comparaison :

- trois petites organisations qui proposent des services informatiques aux entreprises (TI-1, TI-2 et TI-3);

1 L'enquête a été financée par le Fonds québécois de la recherche sur la société et la culture (FQRSC) dans le cadre d'un programme d'Action concertée sur le travail en mutation. 
- deux entreprises de développement de produits d'optique et de télécommunications (Optique I et Optique 2);

- deux bureaucraties :

- le service informatique d'une compagnie d'assurances (Assurances-I),

- et une entreprise de gestion immobilière (Gestion immobilière).

Dans le premier groupe, les organisations comptent entre 100 et 150 employés, alors que dans le deuxième groupe, les organisations en comptent entre 500 et mille. Les employés ne sont syndiqués dans aucune d'entre elles. Toutes ces organisations, dans l'essentiel de leurs activités, transigent constamment avec des clients et empruntent le mode de gestion par projets. On a constitué les services informatiques des deux bureaucraties, suivant une tendance contemporaine répandue, en centres autonomes de services à l'ensemble de l'organisation, chargés de proposer leurs services avec un rendement optimal. Cela étant, ils sont virtuellement en compétition avec des firmes externes, car si ces dernières peuvent proposer les services à un meilleur coût, la direction pourrait décider d'y recourir et d'abolir son service interne d'informatique. En outre, quoique les deux services informatiques de grandes bureaucraties aient été étudiés à des fins de comparaison, leur forme d'organisation du travail est identique, même si le contexte organisationnel diffère.

L'enquête a recueilli en tout le discours de 88 répondants (43 hommes et 45 femmes) entre janvier 2001 et avril 2002, tous occupant des postes liés au génie informatique ou logiciel, en majorité des ingénieurs et des programmeurs; ils ont un niveau élevé de scolarité : $72 \%$ détenaient un diplôme universitaire et $26 \%$ un diplôme d'études collégiales (DEC) ou l'équivalent. Les entretiens en profondeur duraient une heure et demie à deux heures.

\section{LA GESTION PAR PROJETS COMME MODÈLE DE HAUTE PERFORMANCE}

Les professionnels de ce secteur produisent sur mesure des produits complexes et uniques ou 
dispensent des services de même nature. Les cinq PME font affaire avec plusieurs clients externes; les clients des services informatiques des deux bureaucraties sont les autres services de la même bureaucratie. Les cinq entreprises de services technologiques ainsi que les services informatiques des deux bureaucraties organisent le travail par projets, comme l'ensemble du secteur (Berrebi-Hoffmann, 2002; DeFillippi, 2003; Legault, 2005) : chaque projet correspond à un contrat liant l'organisation-fournisseur à une entreprise-client pour lui fournir, bien souvent, à la fois un bien (un produit informatique : logiciel, progiciel) et un service donné (soutien à l'implantation, entretien, service à l'usager). Pour réaliser le produit, on constitue des équipes temporaires, multifonctionnelles, relativement autonomes et composées selon les besoins du client (Alvesson, 1995; Berrebi-Hoffmann, 2002; DeFillippi, 2003, Legault, 2004) qui doivent produire biens ou services à une date et à un prix préétablis par contrat. À la fin de chaque projet, l'équipe est dissoute et les professionnels libérés sont affectés à une autre équipe au terme d'un processus de repêchage qui vise à composer les équipes pour de nouveaux projets (BerrebiHoffmann, 2002; DeFillippi, 2003) ou changent d'emploi.

En soi, bien sûr, l'organisation du travail par projets est vieille comme le monde... Mais chez les informaticiens contemporains, par rapport à leurs prédécesseurs à l'emploi des grandes bureaucraties, par exemple, cela représente un changement d'importance. Il faut d'abord noter que l'histoire des professions de l'informatique est jeune. S'il y a transformation, c'est par comparaison avec un moment où chaque bureaucratie de taille raisonnable avait son service informatique maison et où les PME... n'étaient pas informatisées ou à peine. Les ESTEs ont fleuri parallèlement au mouvement d'informatisation de multiples PME.

\section{Rejet de la sécurité et de la stabilité d'emploi}

Le chef de projet constitue une équipe avec les experts dont les compétences réunies permettront 
de satisfaire la demande du client; ces derniers sont sollicités au besoin et n'ont aucune garantie d'emploi; ils considèrent que leur carrière est nomade et que la mobilité est inhérente à leur profession (Arthur et Rousseau, 1996; Cappelli, 1999; Stewart, 1999; Tremblay, 2003). Chez les professionnels des PME, la mobilité est moins un fléau qu'un atout qu'ils utilisent pour augmenter leur valeur sur le marché du travail, en changeant régulièrement d'emploi pour acquérir de nouvelles compétences, asseoir leur réputation et faire monter les enchères salariales. Ce portfolio de compétences indispensables aux employeurs leur assure le réemploi, dans l'état actuel de la demande. Bref, on ne leur promet ni stabilité d'emploi ni cheminement de carrière mais ils ne le demandent pas non plus :

Company loyalty is a lost virtue. In fact many high-tech employees actually place a stigma on remaining with a company longer than three years; IT pros feel this makes them appear lacking in ambition and thus "unplaceable" (Winn, 2001, p. 63).

Par exemple, chez nos répondants, la stabilité en emploi n'est pas une priorité. En moyenne, dans l'ensemble de l'échantillon, les répondants comptent 4,4 années d'emploi dans leur poste (et pour leur employeur actuel) et 12,2 années d'expérience dans leur type de poste. Cependant, si on distingue les répondants des bureaucraties de ceux des services technologiques, on constate que les répondants des bureaucraties comptent en moyenne 8,7 années d'emploi dans leur poste (et pour leur employeur actuel) alors que ceux des services technologiques en comptent 2,4.

L'employeur des cinq PME ne propose pas de protection contre le risque non plus, ni en termes d'assurances (maladie, invalidité, maternité) ni en termes de formation continue ou de faute professionnelle. Or, la rapide obsolescence des connaissances en informatique contraint ces travailleurs qualifiés à acquérir sans cesse de nouvelles compétences pour conserver leur valeur sur le marché du travail et ils restent chez un employeur tant que ce dernier leur offre l'occasion d'apprendre (Tremblay, 2003), car c'est là leur principal mode de formation. Un autre facteur de 
mobilité de ces employés est la recherche de l'augmentation de leur rémunération, enjeu d'une stratégie de négociation individuelle constante. Leur salaire annuel moyen se situe autour de 60957 \$ CAD en 2000-2001, soit 50 à $100 \%$ de plus que les diplômés de même niveau dans la population générale (Legault, 2004, p. 20-22). Nos répondants se comparent avant tout constamment avec leurs pairs et veillent à ce que leur employeur les rémunère «au niveau du marché ».

Ces professionnels très sollicités échangent un contrat à durée déterminée permettant d'acquérir de nouvelles compétences et de construire sa réputation, une rémunération élevée ainsi qu'une grande autonomie contre leur engagement de court terme à tout faire pour satisfaire au mieux le client d'un projet donné. L'engagement demandé est intense, mais court. Tant le professionnel que l'employeur ne conçoit pas comme une priorité de demeurer longtemps chez le même employeur, au contraire (Legault, 2004; Singh et Vinnicombe, 2000a). Toutefois, cette propension à la mobilité repose sur une grande et constante demande sur le marché du travail (Cappelli, 1999, p. 239). Dans un autre contexte, ces professionnels hésiteraient sans doute avant de quitter leur entreprise. Mais pour l'instant, une grande partie de ces professionnels profitent d'un marché de l'emploi non saturé.

\section{Rejet d'une structure bureaucratique de gestion des ressources humaines}

Dans les sept ESTEs visitées, le service de ressources humaines n'intervient pas dans les relations entre le chef de projet et l'employé (Legault, 2004); ces directions jouent un rôle négligeable dans les décisions qui ailleurs sont de leur ressort, tant en matière d'embauche que de congés, de vacances et d'horaires, se limitant à :

- assurer que l'entreprise se conforme aux principales lois du travail d'ordre public,

- gérer l'application des régimes qu'elle propose (retraite s'il y a lieu, vacances, congés, etc.),

- fournir aux autres services la main-d'œuvre nécessaire aux opérations, 
- procéder aux sanctions, aux licenciements ou aux congédiements lorsqu'on le leur demande.

Lorsque appelé à affecter les professionnels, à établir les horaires, les aménagements des heures de travail, le travail à domicile, les congés et les vacances, le chef de projet décidera le plus souvent seul et de façon très variable, essentiellement selon les exigences du client. Les extraits suivants sont particulièrement éloquents :

Ben y en a...quand quelqu'un est parti de l'équipe, c'est habituellement pas une décision de [1'employeur]. (Ah ça va à ce point-là?) Oui, c'est lui [le client] qui a le dernier mot. C'est lui qui décide qui rentre dans l'équipe, qui décide qui sort : «il fait pas mon affaire, il s'en va ». «Oui, mais attend, on va lui parler » ... Puis c'est arrivé qu'il avait décidé : «Lui s'en va! On veut plus, c'est fini, il est bête au téléphone... », fait qu'ils ont dit : «non, on veut plus ». Fait que là, ils [direction] l'ont averti : «ben dans un mois, tu quittes, tu t'en vas sur un autre projet, là, mais tu quittes cette équipe-là, puis c'est le client » (CGF-1-18-27-8-01-19-3).

Parce que, y a beaucoup de gens, dans notre domaine, c'est le client qui décide. Entre autres, quand on veut des vacances : «oui, mais... on va voir avec le client si y veut bien. » (MF-322-28-3-01-19-3).

Les études révèlent une tendance marquée des ESTEs à gérer sur le mode informel, y compris les ressources humaines (Autier et Picq, 2003; Perrons, 2002; Robertson et O’Malley Hammersley, 2000). Tant les employés que les directions nourrissent une certaine méfiance envers toute formalisation qui pourrait les conduire à un fonctionnement bureaucratique qu'ils associent à une moindre efficacité :

On n'a pas cette lourdeur d'ancienneté, cette lourdeur de bureaucratie, ça, y a pas ça du tout, $\mathrm{du}$ tout, du tout. Mais on a une complexité parce qu'on n'est pas toujours clair, [...]. L'organigramme n'est pas là, sur un mur, avec des cases puis tout le monde sait exactement dans quelle case...Y a un côté un peu gris aux descriptions de tâche de tout le monde, y a un côté un peu gris à l'organigramme... (DSF-13-1-23-8)

Ainsi, les chefs de projet peuvent accorder de façon discrétionnaire des aménagements du temps ou du lieu de travail (Chasserio et Legault, 2005). Ils utilisent ces «faveurs » pour récompenser les comportements de disponibilité et de dévouement et, inversement, ils les refuseront pour sanctionner les comportements moins flexibles. En d'autres termes, le chef de projet les utilise 
comme outils de contrôle et selon le mérite plutôt qu'en fonction des besoins des employés.

De même, les niveaux de rémunération sont établis individuellement. Une part de la rémunération varie en fonction de la performance individuelle, évaluée par le chef de projet pour les employés des deux bureaucraties ainsi que dans trois entreprises de services technologiques (TI 1, TI 2, Optique 1). Quatre entreprises (Assurances-I, TI 1, TI 2, Optique 2) proposent l'option d'achat d'actions à certains employés, selon leur statut, mais dans les organisations visitées, les primes de rendement ne sont pas courantes. La variation des niveaux de rémunération repose essentiellement sur la négociation individuelle, laquelle est fonction de l'estimation de la valeur de l'employé sur le marché, laquelle repose en grande partie sur la satisfaction des précédents clients et employeurs.

\section{La mise à contribution des qualifications et des savoirs}

Pour conserver ou gagner des parts de marchés, les directions doivent mobiliser l'initiative et la créativité des salariés autour d'objectifs de productivité, de qualité, de délais et de satisfaction du client (Gadrey, 2000, p. 202; Anderson-Gough, Grey et Robson, 2000). Toutes les décisions, celles des professionnels comme celles des chefs de projet, sont prises en fonction de la satisfaction du client, dont dépendent à la fois la survie de l'entreprise mais aussi la réputation du professionnel, clé de voûte de son employabilité et de son niveau de rémunération. La réussite des projets fonde la satisfaction des clients qui fonde à son tour l'évaluation des professionnels (Anderson-Gough, Grey et Robson, 2000; Courpasson, 2000; p. 193; Legault et Chasserio, 2003), leur niveau de rémunération et leur réputation, qui est leur meilleur atout sur le marché des emplois. Tout est donc mis en œuvre pour satisfaire la demande unique de chaque client.

Parce que le professionnel, en vertu de son savoir hermétique et du caractère spécialisé de la 
demande, est souvent seul à même de discuter efficacement avec le client, la direction lui transfère le rôle de transiger avec le client pendant la production même et une partie importante de son travail est d'ordre diplomatique (Legault, 2005). Le chef de projet n'est pas l'interlocuteur exclusif du client. Le professionnel est non seulement son interlocuteur principal à plusieurs moments clé mais, en outre, il est souvent appelé à travailler temporairement chez ce dernier. Il doit prendre en compte la demande du client dès le stade de la conception des produits, tenir compte de son évolution et des changements dans les délais de production, produire et rendre à terme un bien au client puis assurer le service après sa production. Il joue un important rôle d'anticipation de la demande du client et de la clientèle en général auprès de la direction, car il est à même de recueillir de précieuses informations.

\section{L'autonomie et l'auto régulation dans l'organisation du travail de ces professionnels}

Loin de rester à l'extérieur de l'entreprise comme le client des entreprises de production de masse, le client des ESTEs est très présent dans le travail quotidien de l'équipe de projet affectée à sa commande, soit en déléguant un représentant auprès des professionnels de l'équipe qui maintient un lien constant, soit en invitant le professionnel à travailler dans son entreprise. Il s'agit là d'un système de contrôle du travail peu coûteux pour l'employeur. Pour garantir l'accès facile du client à l'équipe de projet, les directions mettent en place des structures peu hiérarchisées et une organisation du travail fluide dans lesquelles le contrôle est réduit, et confient ainsi aux professionnels une autonomie opérationnelle considérable pour ce qui est de décider des moyens de réaliser l'objet demandé par le client (Evetts, 1997, Gale et Cartwright, 1995, Cartwright et Gale, 1995). Mis à part un contrôle par le chef de projet quant au respect des budgets et des échéanciers, les professionnels sont libres d'organiser leur relation avec le client et ont un grand pouvoir d'agir (choix de la façon de traiter un problème, hiérarchisation des 
priorités).

Parmi les employés interrogés, 57 \% (38 personnes parmi 67) se disent très, voire totalement, autonomes dans leur poste. $30 \%$ se disent assez autonomes avec un contrôle ponctuel de la part de leur chef de projet, surtout quant au respect des budgets et des délais. En outre, lorsqu'on leur demande quelles sont les qualités de l'employé idéal, $36 \%$ citent l'autonomie comme la première qualité indispensable dans leur profession (Chasserio et Legault, 2008a, 2008b).

Cela se traduit chez les répondants par un extrême souci de respect des délais et des budgets et de la qualité, ainsi qu'en témoignent les expressions suivantes : «livrer dans les délais », «j'appelle cela un succès quand j'ai livré à temps quelque chose qui fonctionne bien », « respecter ses échéanciers » ou encore « rentrer dans les temps du projet et rentrer dans le budget du projet » :

Ben des fois, peut-être que j'ai travaillé un peu plus lentement durant une heure ou deux, pas consciemment peut-être... [...] moi-même, je me mets ... la limite de qu'est-ce que je devrais faire dans la journée. [...]si je ne fais pas assez de choses dans une journée, je ne me sens pas bien! [Est-ce que vous vous sentez coupable?] Oui, je ne sais pas pourquoi. [...] je me sens mal... je vais rester plus longtemps. (STF-13-3-4-4)

Dans un contexte où la satisfaction du client est le principal enjeu de l'évaluation des professionnels et leur principal atout sur le marché mobile des emplois, le contrôle du travail devient superflu car les travailleurs s'auto-disciplinent comme des entrepreneurs, malgré leur statut de salarié (Castells, 2001, p. 55-60; Legault, 2004; Robertson et Swan, 2003). Ils se désignent d'ailleurs souvent comme des consultants, et se perçoivent en position d'entrepreneur sans le nommer (comme l'observent aussi Grugulis, Dundon et Wilkinson, 2000). Plus encore, le chef de projet partage souvent ce pouvoir de contrôle du travail (à des degrés divers) avec le représentant de l'entreprise cliente, dont l'influence sur les conditions de travail est non négligeable: congés, vacances, rémunération des heures supplémentaires, sélection et congédiements... (à ce sujet, lire aussi Anderson-Gough, Grey et Robson, 2000). 
On dira souvent qu'on attribue à ces professionnels un statut d'autonomie responsable (Lincoln et Kalleberg, 1990, p.9, citant Friedman, 1977) dont l'efficacité repose entre autres sur l'importance de la réputation dans un système de carrières nomades, qui induit un contrôle par des normes sociales et professionnelles, intégrées à l'échelle individuelle (Robertson et Swan, 2003). L'adhésion à ces normes, lorsqu'elle fait consensus, permet de résoudre les manifestations de la tension par l'auto-discipline. L'autorité hiérarchique ne serait en effet pas d'un grand secours pour obtenir l'engagement de tels travailleurs. Une telle autonomie met-elle les professionnels à l'abri de la domination? Rien n'est moins sûr.

\section{RHÉTORIQUE D’ENTREPRENEURS... ET DOMINATION INVISIBLE?}

\section{Autonomie et responsabilité, le transfert de l'incertitude et du risque aux employés}

$\mathrm{Au}$ sein de notre population, l'autonomie est tout autant une condition de travail appréciée qu'une exigence d'emploi assortie d'une lourde responsabilité. L'autonomie n'est pas qu'un avantage consenti, c'est aussi une injonction; elle leur est confiée en échange de l'engagement à satisfaire le client. Comme les projets se chevauchent souvent, les employés se voient imposer de considérables demandes concurrentes entre lesquelles ils doivent arbitrer. Ils doivent établir euxmêmes l'ordre de priorités en cas de conflits de tâches, quitte à se voir par la suite reprocher les décisions qu'ils ont prises. Une commande du type : «Débrouillez-vous pour me donner ça à tel moment» ou «Voici l'objectif et organisez-vous » ne caractérise pas l'autonomie décisionnelle qui favorise l'épanouissement mais davantage le stress (Legault et Belarbi-Basbous, 2006). Devant un problème, nos répondants réalisent assez vite qu'ils pourront toujours être tenus responsables du succès ou de l'échec, en vertu même de leur autonomie dans l'établissement des modes opératoires et des priorités. Ces résultats concordent avec ceux d'autres études 
canadiennes réalisées sur de vastes populations industrielles (Godard, 2001). Ces études concluent à la fois à des effets positifs du modèle de haute performance (sentiment d'appartenance et de pouvoir accru, mobilisation, satisfaction au travail) mais aussi à un stress accru et à la réduction des effets positifs lorsque la demande de travail et le niveau de responsabilité augmentent.

Les professionnels sont rarement associés à la négociation des contrats dans lesquels on définit budgets et délais; ils doivent néanmoins par la suite exécuter les travaux qui en découlent en respectant le plus possible les conditions du contrat. Certaines exigences du client sont négociables, mais la plupart sont impérieuses et le professionnel a peu de réel pouvoir de négociation, car les contrats se concluent dans un marché hautement concurrentiel qui confère au client la large part de ce pouvoir (Anderson-Gough, Grey et Robson, 2000). Or, les conditions budgétaires et les délais sont les enjeux clés du risque lié à chaque projet; trop limités, ils favoriseront l'échec du projet. Néanmoins, ce sont les professionnels et les chefs de projet qui en portent la responsabilité. La pression devrait en principe être plus grande dans les cinq PME (qui ont des clients externes) que dans les deux bureaucraties, mais ce n'est pas le cas. Chez Assurances-I, dont les clients sont les autres services de la même organisation, la pression vient de l'intérieur car le service a été converti en centre autonome de profit. Chez Gestion Immobilière, qui emprunte le mode de la gestion par projet, les exigences des clients et le scénario toujours possible de leur départ vers un concurrent exposent les professionnels à la même pression que dans la gestion de projets.

La recherche de la satisfaction du client agit comme un puissant instrument de contrôle, on l'a vu, car c'est là l'enjeu d'une évaluation cruciale. On observe un effet de régulation non négligeable - et peu coûteux - dans la mise en concurrence interne constante des professionnels, 
soit les chefs de projets entre eux et les membres des équipes aussi, désirant une bonne position au prochain repêchage dans l'entreprise pour laquelle ils travaillent actuellement ou dans une autre à court ou à moyen termes, quelquefois une position de chef de projet et le maintien de leur réputation. En conséquence, les membres d'une équipe responsable de l'implantation en trois mois d'un progiciel, par exemple, doivent trancher au quotidien les conflits entre ces impératifs et prendre des décisions logistiques qui, chaque fois, ont une importance déterminante pour le succès ou l'échec du projet. Par exemple, vaut-il mieux sacrifier une phase de test pour respecter les délais, au risque de remettre un produit présentant des bugs, ou privilégier la qualité en prenant son temps, au risque de le voir s'impatienter avant la remise du produit (BerrebiHoffmann, 2002)? Face à l'ampleur du risque, la solution optimale est souvent de tenter de tout faire et, de ce fait, de multiplier les heures supplémentaires pour respecter la date de livraison, ce qu'on peut décrire comme un état de formidable engagement des employés, de même que pour respecter le prix établi, il faut réduire les coûts de production, dont la main-d'œuvre est la principale composante (Chasserio et Legault, 2008a, 2008b). Dans ce contexte, la durée de travail illimitée (sans rémunération des heures supplémentaires) est donc un atout premier pour les directions. Les longues heures de travail caractérisent l'organisation du travail propre aux services informatiques (von Hellens, Nielsen et Trauth, 2001) et à l'économie du savoir (Alvesson, 2000; Bailyn, 1993; Evetts, 1998; Perlow, 1999; Simpson, 1998; Singh et Vinnicombe, 2000a et b). En outre, on y mesure l'engagement des employés, par ailleurs un important facteur de promotion, par le « présentéisme » des employés, soit les nombreuses heures de travail supplémentaires volontiers consenties (Bailyn, 1993, p. 79).

D'autre part, comme c'est au professionnel qu'incombent les fonctions de négociation avec le client, de marketing (analyse-anticipation de la demande), de fidélisation, de positionnement de 
l'entreprise sur un marché, avec des moyens très variables... la responsabilité est énorme. Loin d'influer sur le procédé de production en faisant des représentations auprès de la direction, on retrouve ici le client au centre même du procédé de production, présent à toutes les étapes, négociant chaque étape avec le producteur qui, pour sa part, doit le tenir au courant des obstacles et/ou des possibilités nouvelles qui se présentent au cours des travaux. Dès l'origine, c'est la commande unique même du client qui déclenche le processus de production, plutôt qu'une offre de produit décidée par une direction. La suite des opérations ne fait que renforcer l'influence d'un client non subordonné au fournisseur de services. Le client et le professionnel n'ont pas une relation d'égalité sur le plan de la compétence, mais le contexte de compétition sur le marché donne au demandeur de services un ascendant indéniable. Dans ces organisations, le client pose le cadre même de l'organisation de la production dans l'entreprise et exerce bien des pouvoirs décisionnels ailleurs dévolus à la direction des ressources humaines (Legault et Bellemare, 2008).

Dans les faits, donc, la satisfaction du client est le cadre à l'intérieur duquel s'exerce l'autonomie du professionnel. Par conséquent, la pression aux longues heures de travail est grande et l'autonomie quant à l'aménagement des heures et du lieu de travail est dérisoire, car il y a peu d'ouverture à cet égard dans les ESTEs visitées. Comme la GRH y est peu formalisée, on trouvera peu de politiques ou de mesures officielles d'aménagement du temps ou du lieu de travail; dans l'ensemble des entreprises visitées, seule l'une d'entre elles propose une politique d'aménagement des heures de travail... dont l'application est discrétionnaire selon le chef de projet et le client. On refuse généralement la réduction du temps de travail, notamment le travail à temps partiel, et le travail à domicile dans ces milieux (Legault et Chasserio, 2003). Une chef de projet interrogée dit même :

Moi-même, quand j'ai des gens à 4 jours semaine, je me dis : c'est pas [...] des fonceurs! [...] On a tellement de travail, on pousse les gens, les projets sont pas faciles et tout ça... Pis là, on 
a des gens qui travaillent 4 jours semaine... comme on dit, ça fitte pas [...] On l'offre, je suis contente pour ces gens-là, mais d'un autre côté, des fois, tu regardes ces gens-là, pis t'as peutêtre pas envie de les avoir dans ton équipe. Quand t'as un projet ben urgent... (ASF-3-3-11-701-19-3)

On peut ainsi résumer les obstacles au travail à temps partiel et au refus du travail en heures supplémentaires : les besoins des clients, l'interaction nécessaire entre les membres d'une équipe et les qualifications uniques de chacun dans un processus de production hautement spécialisé et qualifié. Tant les représentants de la direction que les employés (quoique de façon moins uniforme, les hommes adhérant davantage à ces impératifs que les femmes) invoquent ces exigences pour rejeter ces pratiques (Legault et Chasserio, 2006). Ces obstacles ne se comprennent souvent que par la mise en rapport du volume de travail avec les ressources humaines et financières insuffisantes pour réaliser les mandats acceptés (Legault et Chasserio, 2003), quant auxquelles leur statut de salarié ne leur donne aucune emprise.

\section{Importance de la réputation et auto-régulation des professionnels}

Les chefs des projets «de pointe» sont plus exigeants en matière de disponibilité et de compétences et recrutent les plus mobilisés. Pour augmenter leurs chances d'être embauchés dans les projets les plus intéressants, les professionnels les plus ambitieux démontrent disponibilité et dévouement, entre autres en travaillant en heures supplémentaires. Les professionnels qui ne peuvent ou ne veulent pas se prêter au jeu du présentéisme sont cantonnés dans des projets moins intéressants, utilisant des langages informatiques anciens par exemple, ou encore à l'entretien de systèmes informatiques ou au soutien aux usagers (Legault et Chasserio, 2003; lire également Simpson, 1998).

Les travailleurs qualifiés de ce secteur sont davantage soumis au client et à leur équipe de pairs, et sanctionnés par eux, qu'à leur chef de projet. Soucieux au premier chef de leur réputation, nos 
répondants sont disposés à donner la flexibilité et la disponibilité nécessaires au client. La réputation est bâtie, sanctionnée, reproduite et transformée par les décisions des chefs de projets et des clients au moment du réemploi; or, elle est l'atout premier du placement dans un marché de l'emploi caractérisé par une grande mobilité (Berrebi-Hoffmann, 2002, Courpasson, 2000).

Le pouvoir du client induit ainsi l'auto-régulation dans l'équipe et chez l'individu (Legault et Bellemare, 2008) pour produire un milieu exempt d'aménagement ou de réduction des heures de travail, où au contraire une formidable mobilisation des travailleurs les fait travailler en heures supplémentaires souvent non rémunérées (Chasserio et Legault, 2008a, 2008b). On assiste donc à l'intensification du travail sans requérir de surveillance ni de contrôle serré, par l'auto-régulation.

Ainsi, cette chef de projet de la compagnie Assurances-I formule très clairement l'engagement qu'elle attend de ses employés :

Moi, j'attends d'un employé qu'il donne son maximum... au niveau de la productivité. Qu'il puisse s'épanouir dans son travail et à continuer à apprendre... [...] qu'il puisse retrouver une satisfaction personnelle là-dedans, mais qu'il donne son maximum. On est quand même des professionnels, on est très bien rémunérés, alors je m'attends à ce que les gens, quand ils sont au travail, ils donnent leur maximum. (ASF-3-3-11-7-01-19-3)

Les employés sont encouragés à donner «le temps nécessaire » plutôt qu'un temps fixe à leur travail, on les laisse juges en matière de «temps nécessaire », mais chef de projet, client et collègues surveillent leur estimation et l'évaluent.

\section{Engagement élevé par l'adhésion des employés aux objectifs de la direction}

L'évaluation est bien sûr un moment charnière de la progression en carrière, car c'est en général le fondement de la promotion. Or, au moment de l'évaluation, il va de soi qu'on prend en compte «l'engagement» des employés (commitment), tant chez les ingénieurs et les professionnels que chez les cadres en général. De façon uniforme à travers l'ensemble des entreprises de notre 
échantillon, la progression en carrière pour les postes qui nous intéressent est associée à l'engagement (commitment) des employés, telle qu'évaluée par la direction, souvent désignée au cours des entrevues par le terme anglais, même si les entrevues se déroulaient en français.

Il s'agit d'un facteur d'évaluation de première importance, mais sa définition a cependant beaucoup changé au cours des dernières années, à la faveur de la conjoncture économique tributaire de la mondialisation des échanges (Johnson, Lero et Rooney, 2001, p. 30, Stiles, Gratton, Hope-Haily et McGovern, 1997, Rousseau, 1995).

Entre autres, la probabilité de fidélité à long terme envers un employeur perd beaucoup d'importance dans la conjoncture actuelle, au bénéfice des dimensions ponctuelles, liées au rendement de l'employé et à sa contribution à l'entreprise au moment présent (Guest, 1997, 1998; Singh et Vinnicombe, 2000a). Les auteures de cette dernière étude ont interrogé 37 gestionnaires et ingénieurs des deux sexes dans des organisations de haute technologie (aérospatiale) très précisément quant à la notion d'engagement. Les composantes de l'engagement que pointent en priorité les sujets de cette étude ressemblent à celles qu'ont exprimées nos sujets :

- Au premier chef, pour $65 \%$ des personnes interrogées, la livraison du produit (ou l'atteinte de l'objectif) en respectant les délais et le budget impartis (task or objective delivery) et le fait de «se défoncer » au travail, de ne pas hésiter à donner des heures et des efforts supplémentaires (put yourself out, do some extra) (Singh et Vinnicombe, 2000a, p. 238-240). Les femmes insistent davantage sur l'importance de «se défoncer» au travail, sur les efforts qu'il faut mettre à concilier l'emploi et la famille et sur la difficulté de remplir les deux rôles adéquatement (Singh et Vinnicombe, 2000a, p. 240 et 249). Nous y reviendrons plus loin.

- Ensuite, l'implication envers l'organisation et la qualité du travail (59\% des répondants), même si ce n'est pas «à vie » (involvement), se consacrer avec loyauté à l'organisation, en faire un engagement personnel, considérer qu'on représente l'organisation dans ses rapports avec tous ses interlocuteurs : clients, actionnaires, fournisseurs, partenaires et même voisins.

Interrogés quant à l'employé idéal recherché pour occuper les fonctions qui font l'objet de l'enquête, tant les représentants de la direction que les employés mettent en évidence 
principalement trois grandes qualités :

- savoir travailler en équipe (utilisé tant à propos des collègues que des clients),

- faire preuve d'une grande autonomie,

- détenir un ensemble de qualités telles que : satisfaire le client avant tout, demeurer conscient qu'on représente l'entreprise dans tous ses rapports avec les clients, respecter les délais du contrat à tout prix, ne pas compter ses heures, soutenir un stress intense, faire preuve d'engagement (exprimé en général en anglais : commitment).

Nous les avons regroupées sous une même rubrique parce qu'elles ont en commun de témoigner davantage d'un profil d'entrepreneur que d'un profil de salarié, fait dont l'importance se confirme à la lecture des travaux sur les transformations du travail d'ingénieur dans les entreprises de la nouvelle économie. En effet, l'entrepreneur, à la différence du salarié, n'a pas de durée de travail hebdomadaire préétablie par contrat. Il est directement soumis à la concurrence et doit constamment s'assurer de la fidélité du client.

Sur ce dernier point, les données ne peuvent pas être plus éloquentes. Les expressions utilisées par nos sujets sont : «avoir une approche client », «avoir une créativité qui permet de rester compétitif sur le marché », « être à l'écoute des utilisateurs et(ou) des clients », « être disponible 24 heures sur $24 »$, «travailleur 24 heures par jour», « ne pas compter ses heures, donner beaucoup sans rien exiger en retour », «se sacrifier tous ensemble pour faire des heures supplémentaires », « disponible jour et nuit », « dit tout le temps oui, prend tout sur lui », «tenir ses engagements », «disponible à des heures ridicules », etc. Au besoin, travailler à l'étranger pendant plusieurs mois, assurer le soutien permanent au client (demeurer disponible au bout du fil 24 heures par jour) une semaine par mois, implanter de nouveaux systèmes pendant la fin de semaine en sus de sa tâche normale sont des exigences courantes chez nos répondants, dont plusieurs sont de jeunes mères ou pères ou sont sur le point de le devenir. On attend finalement de l'employé un comportement de dévouement, bien plus que d'engagement. Pourtant, il ne 
participe pas aux bénéfices et ne jouit pas de primes ou de bonis, on l'a vu.

La notion d'engagement a beaucoup évolué dans les secteurs du génie; l'importance des critères liés à la satisfaction des exigences des clients augmente et on accorde moins d'importance à la fidélité; on s'attache davantage au fait de se donner comme on se donne à sa propre entreprise, et non comme un salarié «payé à l'heure », comme un «fonctionnaire ", bref à l'entreprenariat chez le personnel embauché. Le discours des ingénieurs salariés ressemble finalement à s’y méprendre à un discours d'entrepreneur. Chez les ingénieurs des entreprises du savoir, on professe que la performance individuelle assure la position compétitive de l'entreprise sur le marché et, par conséquent, la satisfaction du client assure son propre emploi (Singh et Vinnicombe, 2000a).

De même, interrogés quant à leur conception du travail et de la réussite professionnelle, les répondants dessinent ce même profil d'entrepreneur. En effet, les termes suivants reviennent fréquemment :

- satisfaire le client,

- être utile au client et résoudre ses problèmes,

- respecter le délai convenu,

- respecter le budget convenu,

- produire un travail de qualité.

Les discours de nos répondants et ceux de travaux récents sur l'évolution de la notion d'engagement chez les ingénieurs des secteurs de la nouvelle économie concordent quant à l'importance du service à la clientèle dans la nouvelle définition de l'engagement à la faveur d'une nouvelle conjoncture économique. Nous ne prétendons pas à l'existence d'une communion d'intérêts fondamentaux ni à celle d'un consensus touchant tous les aspects de l'organisation du travail. Au contraire, l'expression d'insatisfaction concernant le refus de la réduction du temps de travail, la gestion des heures supplémentaires et de leur rémunération, l'intolérance au travail à 
domicile (Legault et Chasserio, 2003) et les problèmes de santé (Legault et Belarbi-Basbous, 2006) témoigne d'une position critique concernant l'insuffisance de l'effectif embauché pour le travail à faire. Cependant, loin de rechercher une protection auprès de leur employeur contre les demandes des clients, nos répondants imputent à la mondialisation des échanges l'augmentation de la concurrence qui impose aux entreprises de se plier à des exigences très élevées de leur part. Ce mode de fonctionnement génère un stress que les répondants imputent au pouvoir du client et non à leur employeur :

Oui, on évalue toutes les tâches à faire, l'échéancier pour le faire (...). Dans $90 \%$ des cas, le client veut que ça soit deux fois plus vite. Fait qu'on finit toujours par, un peu réduire la période qui serait la période la plus souhaitable. Donc, les gens chez nous qui gèrent les projets, ont du stress parce qu'on leur remet en main un projet qui n'a pas beaucoup de marge de manœuvre par rapport aux délais (DSF-13-1-23-8-01-19-3).

Les longues heures de travail, dont certaines sont gratuites, sont donc acceptées par les répondants comme une contrainte propre au marché. Chez des professionnels indépendants offrant leurs services au sein d'une pratique privée, on ne s'étonnera pas qu'ils soient avant tout soucieux de proposer un service concurrentiel, quel que soit le nombre d'heures nécessaire pour y arriver. Mais chez des salariés privés de la participation aux bénéfices, la chose ne s'explique que par la mobilité des experts sur un marché du travail salarié post-fordiste où la réputation est plus importante que la fidélité à un employeur.

Sans qu'aucune politique de mobilisation ne soit mise en place, les professionnels font preuve d'une formidable mobilisation (Chasserio et Legault, 2008a, 2008b). Ils et elles ressentent un grand engouement pour leur travail, en grande partie en vertu de leur autonomie et de la mise à contribution de leurs qualifications et de leur savoirs.

Le transfert du risque aux employés a cependant pour eux des coûts élevés. L'intensité du travail est très élevée, les heures très longues (Chasserio et Legault, 2005). Ce sont des milieux où le 
risque de problèmes de santé et de sécurité au travail, entre autres les maux liés au stress et à l'épuisement professionnel, est élevé et révèle des contraintes importantes liées à la lourdeur de la responsabilité par rapport aux ressources disponibles pour s'acquitter de la tâche (Legault et Belarbi-Basbous, 2006). Les femmes témoignent enfin d'importants problèmes de conciliation entre la vie privée et la vie professionnelle, qui éloigne les femmes de ce secteur résolument non mixte (Chasserio et Legault, 2008b; Legault et Chasserio, 2006).

\section{CONCLUSION}

L'organisation du travail par projet se répand dans des secteurs d'où elle était absente et c'est cette nouvelle portée, ainsi que son effet d'entraînement, qui en fait un phénomène digne d'intérêt pour les observateurs du marché du travail.

Malgré le rôle très effacé et minimaliste que jouent les directions des ressources humaines dans les ESTEs, on n'observe pas d'anomie dans ces milieux de travail, mais plutôt un mode de régulation stricte et très efficace où le client intervient de très près dans la gestion des ressources humaines, exerce des pressions relayées par l'équipe de pairs et l'individu, dont les intérêts sur le marché du travail sont liés de près à la satisfaction du client.

Bien que n'appartenant pas à l'univers industriel où on observe la mise en place des high consensus / high commitment /high performance environments, on note dans les ESTEs bien des caractéristiques communes à ces environnements de travail.

On remarque entre autres l'apparente perception d'intérêts communs à la direction et aux professionnels, car la satisfaction du client est perçue comme un facteur premier de survie de l'organisation et de maintien de l'employabilité. Ce discours d'entrepreneur n'exprime pas de 
contradiction entre les intérêts des uns et des autres. Par conséquent, dans ces ESTEs, la direction, cadres supérieurs ou chefs de projet qui exercent une autorité déléguée, n'ont pas à transiger avec une contradiction entre l'autonomie conférée aux travailleurs et le contrôle du travail de ces mêmes personnes, alors qu'une telle tension est évoquée au chapitre des problèmes liés aux modèles de haute performance. En effet, les professionnels de ce secteur s'autodisciplinent en vertu des exigences du marché du travail très compétitif au sein duquel leur portfolio, atout premier de l'employabilité, repose sur la satisfaction du client.

La direction n'a pas non plus à transiger avec une contradiction entre la qualité et les coûts, car les exigences de satisfaction du client, reprises à leur compte par les professionnels, les amènent à consentir volontiers à travailler en heures supplémentaires, souvent gratuites (Legault et Chasserio, 2003).

Comme dans le modèle de haute performance, on n’observe pas de réclamation de protection de l'emploi, car la mobilité est acceptée et même on s'en réclame; en outre, la demande pour le travail est constante et élevée. Comme le changement d'emploi est souvent l'occasion d'une augmentation de la rémunération, dans un univers où le niveau moyen de la rémunération dépasse déjà le niveau moyen de la population générale de même niveau de scolarité, la sécurité d'emploi n'est pas une valeur propre à ce milieu, mais bien un repoussoir. Il en va de même de la syndicalisation, qui lorsqu'elle est invoquée par les professionnels (aucune question ne portait sur cet objet dans les entrevues), l'est souvent comme une image repoussoir d'un milieu de travail bureaucratisé auquel on ne veut pas appartenir. Le formalisme, les règles préétablies, l'uniformisation des conditions de travail sont des caractéristiques d'un modèle jugé inapproprié aux services à fournir, que rejettent les professionnels dans ces milieux. 
Ces résultats ne sont pas automatiquement transférables à d'autres milieux de travail, même si on y pratique le travail en équipes, car les ESTEs se caractérisent avant tout par l'importance du rôle régulateur du client et des pairs dans un contexte de très grande autonomie et de très grande mobilité.

En revanche, à notre avis, les résultats présentent de grandes probabilités de généralisation dans les secteurs d'emploi où le travail est géré par projets. Toutefois, le fait que ces travailleurs soient très qualifiés, que leur savoir hermétique les mette à l'abri du contrôle étroit par leurs chefs de projet et rende une partie d'entre eux difficilement remplaçables, ainsi que la très grande demande sur le marché pour leur compétence leur confère un pouvoir sans comparaison, par rapport à leur employeur et sur le marché du travail. Cette combinaison de facteurs favorables doit être prise en compte dans toute tentative de comparaison.

\section{BIBLIOGRAPHIE}

Alvesson, Mats (2000) «Social identity and the problem of loyalty in knowledge-intensive companies », Journal of Management Studies, vol. 37, no 8, p. 1101-1123.

Alvesson, Mats (1995) Management of knowledge-intensive companies, Berlin, Walter de Gruyter.

Anderson-Gough, Fiona, Christopher Grey et Keith Robson (2000) «In the name of the client: The service ethic in two professional services firms », Human Relations, vol. 53, no 9, p. 1151-1174.

Appelbaum, Eilen (2002) «The Impact of New Forms of Work Organization on Workers », dans Gregor Murray, Jacques Bélanger, Anthony Giles et Paul-André Lapointe (dir.), Work employment relations in the high-performance workplace, London, Continuum Press, p. 120-148.

Arthur, Michael B. et Denise M. Rousseau (1996) The Boundaryless Career. A New Employment Principle for a New Organizational Era. Oxford, Oxford University Press.

Autier, Fabienne et Thierry Picq (2003) «Is the resource-based view a useful perspective for SHRM 
research? The case of the video game industry », Communication presented to SASE $15^{\text {th }}$ Annual Meeting on Socio-Economics, Aix en Provence, France, 26-28 juin 2003.

Bailyn, Lotte (1993) Breaking the mold. Women, men, and time in the new corporate world, New York, Free Press.

Bélanger, Jacques, Anthony Giles et Gregor Murray (2002) «Towards a new production model: Potentialities, tensions and contradictions", dans Gregor Murray, Jacques Bélanger, Anthony Giles et Paul-André Lapointe (dir.), Work employment relations in the high-performance workplace, London, Continuum Press, p. 15-71.

Bélanger, Jacques, Paul-André Lapointe et Benoît Lévesque (2002) «Workplace Innovation and the Role of Institutions », dans Gregor Murray, Jacques Bélanger, Anthony Giles et Paul-André Lapointe (dir.), Work employment relations in the high-performance workplace, London, Continuum Press, p. 150180.

Berrebi-Hoffmann, Isabelle (2002) « Nouvelle économie, nouveaux pouvoirs? ». Sciences Humaines, vol. 125 , p. 32-36.

Cappelli, Peter (1999) The New Deal at Work. Boston. Harvard Business School Press.

Carnoy, Martin (2000) Sustaining the New Economy. Work, Family, and Community in the Information Age. New York, Russel Sage Foundation.

Cartwright, Susan et Andrew Gale (1995) «Project management: Different gender, different culture?», Leadership and Organization Development Journal, vol. 16, no 4, p. 12-16.

Castells, Manuel (2001) The Internet Galaxy. Reflections on the Internet Business and Society. New York: Oxford University Press.

Chasserio, Stéphanie et Marie-Josée Legault (2008a) «Strategic human resources management is irrelevant when it comes to highly skilled professionals in the new economy ! », International Journal of Human Resource Management, accepté pour publication

Chasserio, Stéphanie et Marie-Josée Legault (2008b) "Discretionary Power of project managers in knowledge intensive firms and gender issues", Canadian Journal of Administrative Sciences, accepté 
pour publication

Chasserio, Stephanie et Marie-Josée Legault. (2005) «Dans la nouvelle économie, la conciliation entre la vie privée et la vie professionnelle passe par... l'augmentation des heures de travail! ». Recherches Sociographiques, vol. 46, no 1, p. 119-142.

Courpasson, David (2000) L'action contrainte. Organisations libérales et domination, Paris, PUF.

DeFillippi, Robert J. (2003) «Organizational Models for Collaboration in the New Economy ». Human Resource Planning, vol. 25, no 4, p. 7-18.

Edwards, Paul, John Geary et Keith Sisson (2002) « New forms of work organization in the workplace: Transformative, exploitative, or limited and controlled?", dans Gregor Murray, Jacques Bélanger, Anthony Giles et Paul-André Lapointe (dir.), Work employment relations in the high-performance workplace, London, Continuum Press, p. 72-119.

Evetts, Julia (1998) «Managing the Technology but not the Organization: Women and Career in Engineering », Women in Management Review, vol. 13, no 8, p. 283-290.

Evetts, Julia (1997) «Women and careers in engineering: Management changes in the work organization », Women in Management Review, vol. 12, no 6, p. 228-233.

Friedman, A. (1977) Industry and Labor, London, Macmillan.

Gadrey, Jean (2000) Nouvelle économie, nouveau mythe?, Paris, Flammarion

Gale, Andrew et Susan Cartwright (1995) « Women in project management: Entry into a male domain? », Leadership and Organization Development Journal, vol. 16, no 2, p. 3-8

Giles, Anthony, Gregor Murray et Jacques Bélanger (2002) «Introduction», dans Gregor Murray, Jacques Bélanger, Anthony Giles et Paul-André Lapointe (dir.), Work employment relations in the high-performance workplace, London, Continuum Press, p. 1-14.

Godard, John (2001) "High Performance and the Transformation of Work? The Implications of Alternative Work Practices for the Experience and Outcomes of Work", Industrial and Labor Relations Review, vol. 54, no 4, p. 776-805.

Grugulis, Irena, Tony Dundon et Adrian Wilkinson (2000) «Cultural control and the culture manager: 
Employment practices in a consultancy », Work, Employment and Society, vol. 14, no 1, p. 97-116.

Guest, David E. (1998) «Beyond HRM: Commitment and the contract culture», dans Sparrow, P. et Marchington, M. (dir.), Human Resource Management: The new agenda, London, FT Pitman Publishing, p. 37-51.

Guest, David E. (1997) «Human resource management and performance: A review and research agenda », International Journal of Human Resource Management, vol. 8, no 3, p. 263-276.

Johnson, Karen L., Donna S. Lero et Jennifer A. Rooney (2001) Recueil travail-vie personnelle 2001, 150 Statistiques canadiennes sur le travail, la famille et le bien-être, Développement des ressources humaines Canada et Center for Families, Work and Well-Being

Legault, Marie-Josée et Guy Bellemare (2008) "Emergent modes of labour regulation in management-byproject processes and theoretical issues for industrial relations", Relations industrielles -Industrial Relations, accepté pour publication

Legault, Marie-Josée et Stéphanie Chasserio (2006) «La gestion de projets dans les services technologiques aux entreprises et ses effets de genre », Regards sur le travail, vol. 2, no 3 (Revue du ministère du travail du Québec, comité d'évaluation par les pairs), accessible en ligne : http://www.travail.gouv.qc.ca/actualite/regardstravail/regardstravail-vol02-03.pdf

Legault, Marie-Josée et Hind Belarbi-Basbous (2006) «Gestion par projets et santé mentale au travail dans la nouvelle économie », Perspectives interdisciplinaires sur le travail et la santé (PISTES), vol. 8, no 1 , accessible en ligne :

www.unites.uqam.ca/pistes

Legault, Marie-Josée (2005) «Differential Gender effects of project management and management by project on skilled professionals », dans Kay S. Devine et Jean-Noël Grenier (dir.), Reformulating Industrial Relations in Liberal Market Economies - Reformuler les relations industrielles dans une économie de marché libérale, Actes du $41^{\mathrm{e}}$ colloque de l'Association canadienne des relations industrielles (ACRI) - Canadian Industrial Relations Association (CIRA), Concord (Ontario), ACRICIRA et Captus Press, p. 105-124. 
Legault, Marie-Josée, avec la collaboration de Stéphanie Chasserio. (2004) Les politiques et les pratiques de conciliation entre la vie professionnelle et la vie privée dans sept organisations de la nouvelle économie de Montréal. Remis au Fonds québécois de recherche sur la société et la culture (FQRSC), 120 pages. http://www.fqrsc.gouv.qc.ca/recherche/index1.html

Legault, Marie-Josée et Stephanie Chasserio (2003) «Family obligations or cultural constraints? Obstacles in the path of professional women ». Journal of International Women Studies, vol. 4, no 3, p. 108-128.

http://www.bridgew.edu/soas/jiws/May03/Family_Obligations.pdf

Legault, Marie-Josée (1988) «Le métier de chercheur scientifique en sciences sociales et la sociologie des professions », Sociologie et sociétés, vol. 20, n², p. 163-176

Lincoln, James R. \& Kalleberg, Arne L. (1990) Culture, control and commitment. Study of work organization and work attitudes in the United States and Japan, Cambridge, Cambridge University Press.

Linhart, Danièle (2004) La modernisation des entreprises. Collection Repères. Paris, Éditions La Découverte.

Martuccelli, Danilo (2001) Dominations ordinaires. Explorations de la condition moderne, Paris, Éd. Balland

Murray, Gregor, Jacques Bélanger, Anthony Giles et Paul-André Lapointe (2002) Work Employment Relations in the High-Performance Workplace, London, Continuum

Périlleux, Thomas (2001) Les tensions de la flexibilité. L'épreuve du travail contemporain. Paris, Desclée de Brouwer.

Perlow, Leslie A (1999) «The Time Famine: Toward a Sociology of Work Time», Administrative Science Quarterly, vol. 44, p. 57-81.

Perrons, Diane (2002) «Gendered divisions in the new economy: Risks and opportunities ». GeoJournal, vol. 56 , no 4 , p. 271-280.

Robertson, Maxine et Jacky Swan (2003) «Control - What control? Culture and ambiguity within a 
knowledge intensive firm ». Journal of Management Studies, vol. 40. no 4, p. 831-859.

Robertson, Maxine et Geraldine O’Malley Hammersley (2000) «Knowledge management practices within a knowledge-intensive firm: The significance of the people management dimension ». Journal of European Industrial Training, vol. 24, nos 2/3/4, p. 241-252.

Rousseau, Denise M. (1995) Psychological contracts in Organizations: Understanding Written and Unwritten Agreements, Thousand Oaks, Sage.

Rousseau, Denise M. et Martin M. Greller (1994) « Human Resources Practices: Administrative Contract Makers ». Human Resources Management, vol. 33, no 3, p. 385-401.

Simpson, Ruth (1998) « Presenteeism, Power and Organizational Change: Long hours as a Career Barrier and the Impact on the Working Lives of Women Managers ». British Journal of Management, vol. 9, Special Issues, no 1, p. 37-50.

Singh, Val et Susan Vinnicombe (2000a) «What does "commitment" really mean? Views of UK and Swedish engineering managers ». Personnel Review, vol. 29, no 2, p. 228-258.

Singh, Val et Susan Vinnicombe (2000b) "Gendered Meanings of Commitment from High Technology Engineering Managers in the United Kingdom and Sweden", Gender, Work and Organisation, vol. 7 , no 1 , p. $1-19$

Stewart, Thomas A. (1999) Intellectual Capital. The New Wealth of Organizations. New York: Doubleday Editions.

Stiles, Philip, Lynda Gratton, Veronica Hope-Haily et Patrick McGovern (1997) «Performance management and the psychological contract », Human Resource Management Journal, vol. 7, no 1, p. $57-66$

Tremblay, Diane Gabrielle (2003) « Nouvelles carrières nomades et défis du marché du travail; une étude dans le secteur du multimédia ». Revue de Carriérologie, vol. 9 no 1-2, p. 255-280.

Von Hellens, Liisa A., Sue H. Nielsen et Eileen M. Trauth (2001) «Breaking and entering the male domain. Women in the IT industry », dans Mark Serva (dir.), Proceedings of the 2001 Association for computing machinery-Special Interest group on computer personnel research (ACM-SIGCPR) 
Conference, San Diego, p. 116-131.

Winn, John (2001) «Techies in demand ». Boardwatch Magazine, vol. 15, no 5, p. 63-67.

INTRODUCTION.....................................................................................................................................................

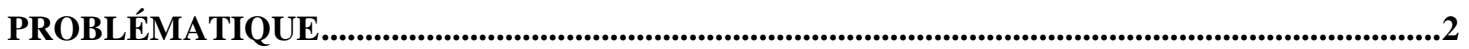

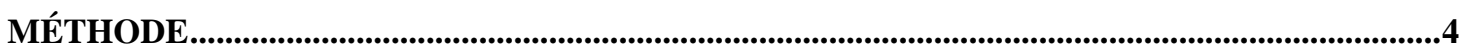

LA GESTION PAR PROJETS COMME MODÈLE DE HAUTE PERFORMANCE ........................5

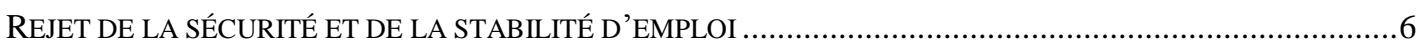

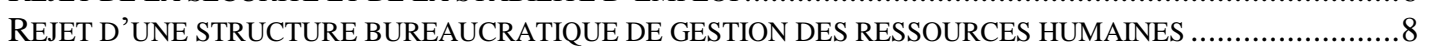

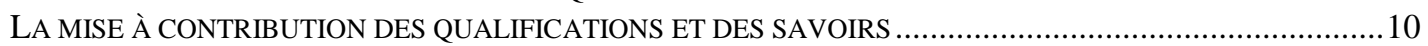

L'AUTONOMIE ET L'AUTO RÉGULATION DANS L'ORGANISATION DU TRAVAIL DE CES PROFESSIONNELS11

RHÉTORIQUE D'ENTREPRENEURS... ET DOMINATION INVISIBLE? ...............................13

AUTONOMIE ET RESPONSABILITÉ, LE TRANSFERT DE L'INCERTITUDE ET DU RISQUE AUX EMPLOYÉS ...13

IMPORTANCE DE LA RÉPUTATION ET AUTO-RÉGULATION DES PROFESSIONNELS ..................................17

ENGAGEMENT ÉLEVÉ PAR L'ADHÉSION DES EMPLOYÉS AUX OBJECTIFS DE LA DIRECTION .....................18

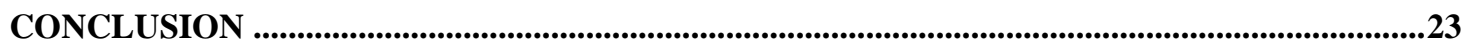

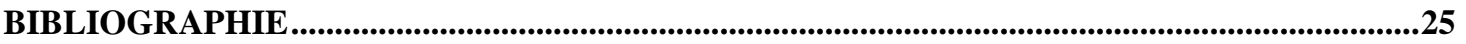

